\title{
神经退行性疾病的光学疗法及其应用展望
}

\author{
魏勋斌 ${ }^{1,2}$, 周非凡 ${ }^{3}$, 陶乐婵 ${ }^{2}$, 杨思华 ${ }^{4}$, 顾波波 ${ }^{2}$ \\ (1. 北京大学跨学部生物医学工程系, 北京 $100191 ； 2$. 上海交通大学生物医学工程学院 Med-X 研究院, 上海 200030; \\ 3. 深圳大学物理与光电工程学院, 广东深圳 $518061 ; 4$. 华南师范大学生物光子学研究院 \\ 暨激光生命科学教育部重点实验室, 广州 510631)
}

\begin{abstract}
摘要: 神经退行性疾病是由神经元结构或功能逐渐丧失导致认知及运动障碍的一类不可逆损伤性疾病, 目前尚无安全有效的 治疗方法。探索无创的物理治疗手段在神经退行性疾病中的应用潜力, 对疾病缓解与有效控制具有重大的意义。光学疗法是 利用光线与组织的相互作用, 通过光化学或光物理反应治疗疾病和促进机体康复的方法, 具有精准性和微创性的技术特点。 其中, 弱光治疗作为一种无创光疗类型, 在促进伤口愈合、缓解疼痛、炎症消退、组织再生等方面已广泛应用于临床。临床 研究也证实弱光治疗能够有效改善神经退行性疾病病患的病理症状, 作为一种无创物理疗法, 弱光治疗为神经退行性疾病的 缓解和有效控制提供了非常具有前景的新方向。本文综述了弱光治疗在神经退行性疾病中的研究进展, 并结合光电子技术的 发展展望其应用前景, 研究提出: 需阐明弱光作用机理及量效关系, 开发新型弱光治疗技术, 完善临床验证体系及评价指标, 尽快造福病患，服务社会。
\end{abstract}

关键词: 光学疗法; 弱光治疗; 无创疗法; 神经退行性疾病

中图分类号: R741 文献标识码: A

\section{Phototherapy of Neurodegenerative Diseases: Mechanism, Application, and Prospect}

\author{
Wei Xunbin ${ }^{1,2}$, Zhou Feifan ${ }^{3}$, Tao Lechan ${ }^{2}$, Yang Sihua ${ }^{4}$, Gu Bobo ${ }^{2}$ \\ (1. Biomedical Engineering Department, Peking University, Beijing 100191, China; 2. Med-X Research Institute \& School of \\ Biomedical Engineering, Shanghai Jiao Tong University, Shanghai 200030, China; 3. College of Physics and Optoelectronic \\ Engineering, Shenzhen University, Shenzhen 518061, Guangdong, China; 4. MOE Key Laboratory of Laser Life Science \& \\ Institute of Laser Life Science, College of Biophotonics, South China Normal University, Guangzhou 510631, China)
}

\begin{abstract}
Neurodegenerative diseases are a heterogeneous group of irreversible illnesses caused by the progressive loss of neuronal structure or function, leading to cognitive and movement disorders, without safe and effective treatments. Exploring the application potential of non-invasive physical therapy for neurodegenerative diseases is of great significance for disease remission and effective control. Phototherapy is a method that uses the interaction of light and tissue to treat diseases and promote the rehabilitation of the body through photochemical or photophysical reactions, with precise and minimally invasive features. Low-light therapy is a type of non-invasive phototherapy that is used in promoting wound healing, pain relief, inflammation regression, and tissue regeneration. Clinical studies have also confirmed that low-light therapy can effectively improve the pathological symptoms of patients with neurodegenerative diseases. Thus, as a non-invasive physical therapy, low-light therapy provides a promising new direction for the
\end{abstract}

收稿日期 : 2020-03-25; 修回日期 : 2020-04-28

通讯作者: 魏别斌, 北京大学跨学部生物医学工程系教授, 主要研究方向为生物医学工程; E-mail: xwei@bjmu.edu.cn

资助项目：中国工程院咨询项目 “我国激光技术与应用 2035 发展战略研究” (2018-XZ-27)

本刊网址 : www.engineering.org.cn/ch/journal/sscae 
relief and effective control of neurodegenerative diseases. This article summarizes the research progress of low-light therapy for neurodegenerative diseases and prospects its application in conjunction with the development of optoelectronic technology. It is suggested that the mechanism and dose effect relationship of low light should be clarified, new low-light treatment technology should be developed, and the clinical verification system and evaluation index should be improved, so as to benefit patients and serve the society as soon as possible.

Keywords: phototherapy; low-light therapy; non-invasive physical therapy; neurodegenerative diseases

\section{一、光学疗法的兴起}

神经退行性疾病是机体神经元结构或功能逐 渐丧失导致认知及运动障碍的一类不可逆损伤性 疾病, 包括肌萎缩侧索硬化症 (ALS)、帕金森病 $(P D) 、$ 阿尔茨海默病 $(A D)$ 、亨廷顿氏病 $(H D)$ 以及脊髓性肌萎缩症（SMA）等。由于目前发病机 制尚不明确, 以及缺乏有效的治疗手段, 神经退行 性疾病已成为基础科学和临床科学的研究焦点。每 年，全世界被诊断患有与年龄相关的神经退行性疾 病的患者数量正在迅速增加, 并逐渐成为老龄化人 口中常见的死亡原因。 $\mathrm{AD}$ 和 $\mathrm{PD}$ 是最常见的两种 神经退行性疾病, 我国 65 岁以上老年人 PD 的发病 率为 $1.7 \%$, 患者人口已经超过 250 万, $\mathrm{AD}$ 患病率 为 $3.21 \%$, 患病人口已经超过 800 万。随着中国人 口老龄化的迅速发展, $\mathrm{AD}$ 和 PD 发病率逐年攀升, 不仅威胁患者自身的生活质量，而且其不断增加的 医疗和护理费用已造成严重的社会经济困境。然而, 由于目前还没有有效的治疗方法来阻止或延缓患者 病情的恶化, 因此 $\mathrm{AD}$ 和 PD 的治疗和护理将面临 巨大挑战。开发早期无创检测技术和无创治疗手段 是预防和控制该类疾病发展、缓解社会医疗负担的 关键所在。然而, 神经疾病的无创诊疗一直是个世 界性难题。

光学疗法是一种利用光线照射损伤部位从而缓 解或治疗疾病的一种无创或微创治疗手段。1903 年, 丹麦科学家 Niels Finsen 凭借红光治疗红斑狼疮获 得第三届诺贝尔生理学或医学奖, 自此开启了光学 治疗的新篇章。1967 年匈牙利科学家 Endre Mester 发现 $694 \mathrm{~nm}$ 红宝石激光可以促进小鼠毛发再生和 伤口愈合, 这是首次发现的激光生物刺激效应, 它 开启了医学领域的一条崭新的途径, 并进而发展形 成 “低剂量激光疗法”, 又称弱激光治疗, 是指激 光作用于生物组织时, 不造成生物组织不可逆的损 伤, 但可以刺激机体产生一系列的生理生化反应,
对组织或机体起到调节、增强或抑制的作用, 从而 达到治疗疾病的目的。目前, 光学疗法形成了包括 强激光治疗、光动力治疗、弱激光治疗在内的三大 光学治疗技术，已广泛应用于临床多个科室多种疾 病的治疗, 并以精准性、微创 / 无创性为技术突破 方向, 引领医学治疗模式的转变。

近年来, 许多研究利用弱光治疗动物神经退行 性疾病, 发现可通过调控神经元及小胶质细胞活性, 有效减缓神经元死亡, 延缓疾病进程 $[1,2]$ 。相关临 床试验也获得积极结果, 能够有效改善神经退行性 疾病患者的病理症状。基于光电子技术的迅猛发展 和光遗传技术的应用, 弱光治疗设备逐渐微型化、 智能化, 弱光治疗技术趋向于精准靶向调控。作为 一种副作用小的无创物理疗法, 弱光治疗为神经退 行性疾病提供了一个非常具有前景的新方向。探讨 弱光治疗在神经退行性疾病治疗中的潜在应用和发 展, 将引领其发展方向, 促进其临床应用, 规范其 市场转化, 为神经退行性疾病患者提供有效的治疗 方法, 为缓解社会老龄化导致的护理和医疗负担提 供可行的解决方案。

\section{二、弱光治疗发展现状}

弱光治疗, 又称光生物调节 $(\mathrm{PBM})$, 是使用 红光或近红外光调节受损组织达到治疗目的的一种 无创光疗类型, 在促进伤口愈合、缓解疼痛、炎症 消退中有良好功效, 已广泛应用于临床。尽管已有 系列研究报道 PBM 在神经保护中的作用, 但其光 生物学机制尚不完全清楚。美国 Hamblin 教授 $[3,4]$ 团队的研究表明 $810 \mathrm{~nm}$ 弱激光可通过上调神经元 细胞色素 $\mathrm{C}$ 氧化酶 ( $\mathrm{CCO}$ ) 活性, 进而增强线粒 体功能, 增加腺嘌呤核苷三磷酸 (ATP) 产量, 从 而缓解神经元死亡。国内邢达教授团队多年的研究 认为 $633 \mathrm{~nm}$ 弱激光通过激活细胞膜表面非受体酪 氨酸激酶, 从而引发一系列下游信号转导, 增强细 
胞增殖信号, 抑制细胞调亡信号, 从而抵抗神经元 死亡 [5 7]。另外, 其团队发现弱激光可以通过激 活非受体酪氨酸激酶来抑制活化的小胶质细胞, 降 低其产生的神经毒性, 并增强其吞噬能力, 清除 $\beta$ 淀粉样蛋白 (A $\beta$ ) 聚集体 [8]。两种作用机制如图 1 所示。目前, 越来越多的研究小组尝试使用不同波 长、不同模式的光调控神经细胞活性, 达到延缓疾 病进展的目的。

\section{（一）弱光治疗与阿尔兹海默症}

$\mathrm{AD}$ 是一种以进行性认知功能障碍为临床特征 的慢性神经系统退行性疾病。病症主要表现为: 记 忆与认知障碍、情绪不稳定、机能丧失等。病理特 征主要是 $A \beta$ 的异常聚集和神经元纤维缠结(NFTs), $A \beta$ 和 NFTs 具有的细胞毒性会造成神经元死亡, 进 一步导致认知障碍。目前, 药物治疗是 $\mathrm{AD}$ 治疗的 主流方法, 通过美国食品药品监督管理局 (FDA) 认证的 $\mathrm{AD}$ 治疗药物一共有五种, 其中达克林（Tacrine）由于副作用太大已经撤市, 剩余四种为加兰他 敏 (Galantamine)、利斯的明 (Rivastigmine)、多奈 哌齐（Donepezil）和美金刚（Memantine）, 可以一 定程度缓解 $\mathrm{AD}$ 导致的认知与记忆障碍, 但是不能 治愈 $\mathrm{AD}$, 并且部分患者用药后会产生呕吐、头痛、 幻觉等副作用 $[9,10]$ 。因此无副作用、疗效显著的 治疗手段是当前研究的热点与难点。近年来, 国内
外临床前及临床研究均证实弱光疗法能够有效改善 $\mathrm{AD}$, 有望发展为一种针对 $\mathrm{AD}$ 的新的物理疗法。 在针对 $\mathrm{AD}$ 动物模型的治疗中发现, 弱光能够 显著降低大脑皮质和海马中 $\mathrm{A} \beta$ 斑块体积和数量, 有效缓解 $\mathrm{AD}$ 症状。Taboada 等 [11] 利用 $808 \mathrm{~nm}$ 连 续激光经颖 PBM (tPBM) 对 A $\mathrm{PPP}$ 模型小鼠进行 6 个月的照射治疗, 结果表明 tPBM 可以改善水迷 宫实验的表现, 减少脑部、脑脊液和血浆中 $\mathrm{A} \beta$ 水 平, 减少脑部炎症因子白细胞介素 $1 \beta$ (IL-1 $\beta$ ), 肿 瘤坏死因子 $\alpha(\mathrm{TNF}-\alpha)$ 含量, 改善线粒体功能, 增加耗氧量和 ATP 产量, 增加 c-Fos 蛋白, 改善神 经元功能。同时发现 $\mathrm{tPBM}$ 疗效与功率密度相关, 该实验中功率密度为 $50 \mathrm{~mW} / \mathrm{cm}^{2}$ 时, 疗效最好, 过 高或过低的功率密度疗效均不显著。Tao 等人 [12] 利用 $1070 \mathrm{~nm}$ LED 对 APP/PS1 小鼠进行 40 天的照 射治疗, 结果表明光照组小鼠水迷宫表现明显改善, 海马区 $\mathrm{A} \beta$ 斑块面积明显减少。Purushothuman 等 [13] 发现 PBM（670 nm, LED）治疗 APP/PS1 模型小鼠 同样可以减少海马、皮层和脑干的 $\mathrm{A} \beta$ 斑块水平。 同时, 可以减少 $\mathrm{K} 3$ 模型小鼠海马、皮层和脑干的 NFTs, 改善氧化应激的损伤。Farfara 等 [14] 发现 PBM (670 nm 或 $904 \mathrm{~nm}$ ) 照射四月龄 5XFAD 小鼠, 骨髓间充质细胞分化为单核细胞的能力增强, 吞噬 可溶性 $\mathrm{A} \beta$ 的活性增强, 从而减少脑内 $\mathrm{A} \beta$, 提高小 鼠的认知功能和空间学习能力。

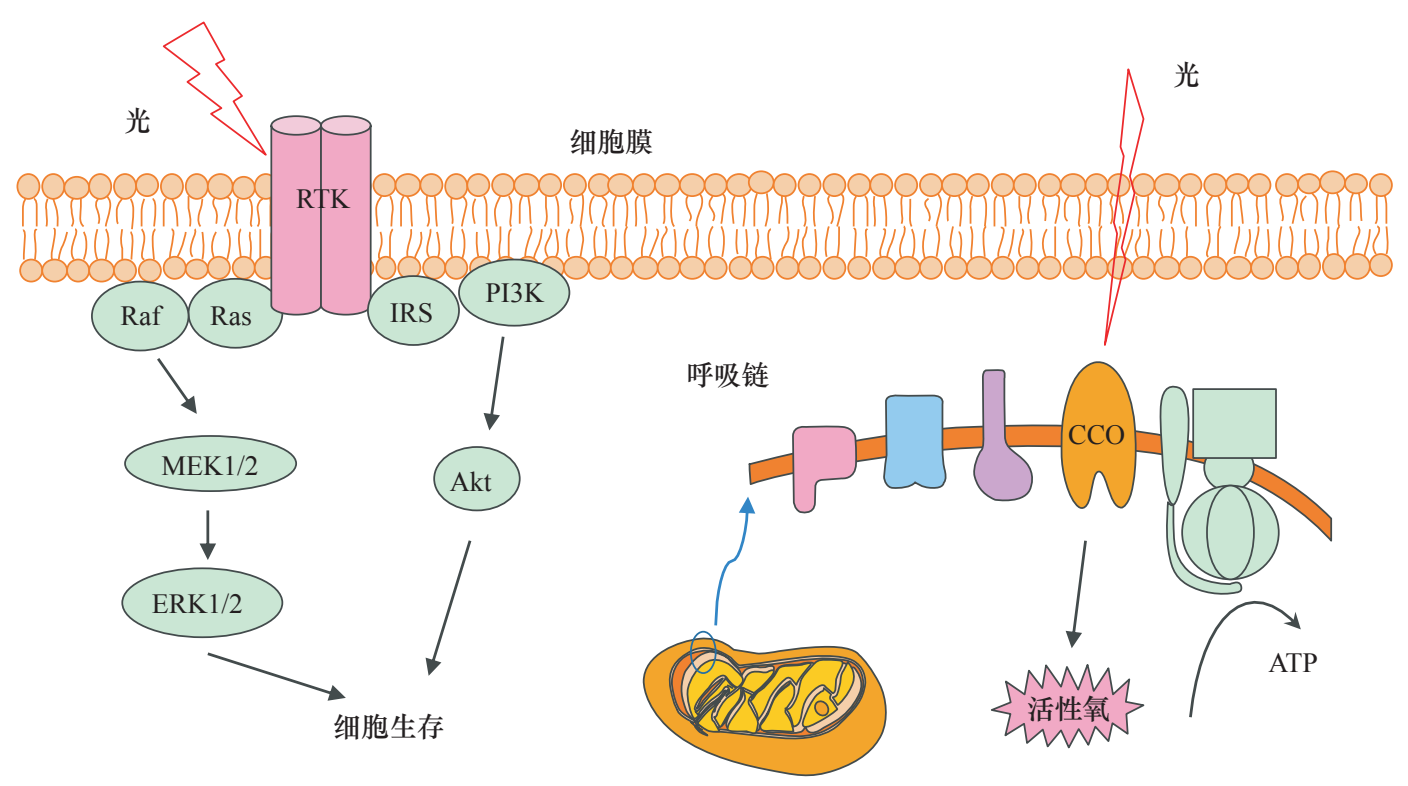

图 1 PBM 作用于细胞的潜在机制 
弱光治疗除了能降低 $\mathrm{AD}$ 模型小鼠大脑中 $\mathrm{A} \beta$ 水平以外, 还能有效减少 $\mathrm{A} \beta$ 诱发的一系列神经损 伤。 $\mathrm{A} \beta$ 和 tau 低聚物与突触结合而导致的突触功能 障碍是 $\mathrm{AD}$ 疾病发生发展过程中最早出现的损伤之 一。利用 PBM $(670 \mathrm{~nm})$ 可以减少 $\mathrm{Tg} 2576$ 模型小 鼠海马区、皮层中突触与 $\mathrm{A} \beta$ 低聚物的结合, 增加 突触线粒体膜电位, 从而改善突触功能障碍、长时 程增强损伤 [15]。除此之外, 利用 PBM (808 nm) 同样可以减少 $A \beta$ 诱导的神经毒性。该小组在大鼠 海马区注射 $\mathrm{A} \beta_{1-42}$, 随后对大鼠进行 PBM 治疗, 结 果表明 PBM 可以改变分裂和融合蛋白水平, 从而 恢复线粒体动力学, 抑制 $\mathrm{A} \beta$ 引起的线粒体碎片化; 调节 Bax/Bcl-2 水平和线粒体抗氧化物表达从而恢 复线粒体内稳态，抑制 $\mathrm{A} \beta$ 诱导的线粒体膜电位损 伤, 促进 CCO 活性和 ATP 合成。同时, PBM 还可 以抑制 $\mathrm{A} \beta$ 诱导的 6-磷酸葡萄糖脱氢酶 (G6PDH) 和还原型辅酶 II（NADPH）氧化酶活性, 减少氧 化的线粒体 DNA 和过度自噬作用, 增强海马 CA1 神经元的抗氧化能力, 减少氧化损伤; 并抑制 $A \beta$ 诱导的反应性胶质化、炎症和过磷酸化 [16]。

除了常用的红光和近红外波段连续光治疗模 式, 其他波段和照射模式也能实现传统弱光诱导 的生物学效应, 但其光感器或相应的机制尚不清

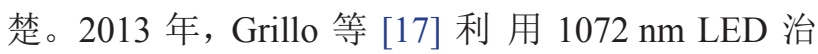
疗 TASTPM 转基因痴呆模型小鼠可减少齿状回 A $\beta$ 斑块水平。除此之外, PBM 明显增多热休克蛋白 (HSPs) HSP60、HSP70、HSP105、HSP27 等，同 时下调 $\mathrm{HSP} \alpha \mathrm{B}$-crystallin 水平来减少 $\mathrm{A} \beta$ 蛋白毒性, 推测其可能诱导热弛豫过程, 局部释放热能, 从 而改变细胞内信号转导机制。同时, Zinchenko 等 人 [18] 发现, $\operatorname{tPBM}\left(1267 \mathrm{~nm}, 32 \mathrm{~J} / \mathrm{cm}^{2}\right)$ 可以通过 刺激大脑和颈部的淋巴循环系统来促进淋巴循环对 $\mathrm{A} \beta$ 的清除。细胞内 $\mathrm{A} \beta$ 会抑制皮层雉体细胞的大电 导钙离子激活钾通道活动 ( $\mathrm{BK}$ ), 从而使动作电位 峰宽增加。宽峰动作电位会增加䥻离子内流, 从而 导致内质网应激。Zhang Y 教授 [19] 等利用可见光 (2 Hz) 刺激 3xTg AD 模型小鼠, 结果表明, 可见 光刺激并不改变小鼠扣带回皮层的 $\mathrm{A} \beta_{42}$ 水平, 但是 可以通过上调扣带回和外侧杏仁核的同源蛋白 $1 \mathrm{a}$ 水 平来改善 $\mathrm{A} \beta$ 对雉体细胞 $\mathrm{BK}$ 通道抑制作用, 从而 改善小鼠空间学习能力和条件恐惧记忆。2016 年, 《自然》(Nature) 发表 Li-Huei Tsai 教授团队研究文
章, 发现 $40 \mathrm{~Hz}$ LED 白光可引起脑部视觉皮层区域 无创地产生伽马震荡, 促使 $\mathrm{AD}$ 小鼠大脑的主要免 疫细胞小胶质细胞改变形态, 进而增加对淀粉样蛋 白的吞噬, 从而缓解 AD 疾病 [20]。2019 年, 其团 队对 $40 \mathrm{~Hz}$ 光照治疗的机理进行探究, 发现这种光 照方式是通过改变一些与神经和突触损伤、小胶质 形态相关的基因表达来减少小鼠脑部的神经和突触 损伤, 减少小胶质细胞激活从而减少神经炎症 [21]。 同年, 该团队对 $40 \mathrm{~Hz}$ 视觉刺激和 $40 \mathrm{~Hz}$ 听觉刺激 联合治疗的效果进行了探究, 发现联合刺激同样 可以减少小鼠脑部的 $A \beta$ 、减少激活的小胶质细胞、 星形胶质细胞, 改善认知与记忆能力 [22]。事实上, $\mathrm{PBM}$ 似乎有能力从几个不同的角度瞄准 $\mathrm{AD}$, 使其 成为一种可行的潜在治疗方法。

光疗能够有效缓解 $\mathrm{AD}$ 的病理症状已在临床试 验中得到证实。周畅课题组用 $670 \mathrm{~nm}$ 红光对轻度 认知障碍患者进行鼻腔内照射治疗, 能够降低患者 血清内 $A \beta$ 含量, 影响 $A \beta$ 代谢过程, 延缓疾病发 展 [23]。美国 Arakelyan 教授对 154 位 $\mathrm{AD}$ 病人分别 进行 PBM、磁场疗法 (MFT)、光色疗法 (LCT)、 $\mathrm{AD}$ 药物疗法和 PBM+MFT+LCT 结合疗法五种疗法, 并对其疗效进行比较。结果表明, 进行组合疗法、 PBM、MFT 或药物疗法的病人其阿尔茨海默病评估 量表 (ADAS-cog) 分数相较于安慰剂组均明显下降, 其中组合疗法效果最显著, PBM 疗法其次, 随后是 药物疗法, MFT 效果最差。该研究认为 PBM 是疗 效仅次于组合疗法的一种治疗 $\mathrm{AD}$ 的方法, 同时组 合疗法也是一个具有前景的治疗方式 [24]。Maksimovich [25] 对比血管内 PBM (可见光, $20 \mathrm{~mW}$, 连 续/脉冲）治疗的患者（46 例）和药物 Memantin、 Rivastigmine 治疗的患者 (43 例) 的疗效差异, 结 果表明 PBM 组的脑微循环和代谢得到改善, 使得 痴呆症永久性减轻以及认知恢复。Lim [26] 通过 $810 \mathrm{~nm}$ 脉冲 LED 和 $633 \mathrm{~nm}$ 连续 LED 治疗 4 名 $\mathrm{AD}$ 患者, 在一年的观测中, 四名患者认知能力均显著 提高, 其中一人甚至从基线 “显著认知障碍” 提升 到了实际上的 “无认知损害” 状态。Berman 等 [27] 对 11 位轻度认知障碍病人进行小型双盲实验, 8 位 病人进行 $1070 \mathrm{~nm}$ 光照治疗, 3 位病人作为安慰剂 组。在连续的 28 天治疗后, 治疗组病人执行能力 (时 钟绘制、即时回忆、练习记忆、视觉注意力和任务 切换）均有一定程度改善。脑电图（EEG）结果显 
示治疗组病人警觉性、注意力有改善, 焦虑情绪得 到缓解。Saltmarche 等[28] 通过 $810 \mathrm{~nm}, 10 \mathrm{~Hz}$ 经 颖骨照射和鼻腔内照射结合, 治疗 5 位痴呆症病人, 12 周后参与者表现出明显的改善 (精神状态测试评 分平均提高 5 分), ADAS-cog 分数也有明显提高。 同时, 参与者表示焦虑和愤怒情绪减少, 睡眠质量 提高, 且没有观察到不良反应。

\section{（二）弱光治疗与帕金森病}

PD 是一种运动障碍类神经退行性疾病, 平均 发病年龄为 60 岁左右。患者大脑黑质纹状体多巴 胺神经细胞随着时间的推移逐渐死亡, 导致静止性 震颤和肢体僵硬。对于 PD 患者, 有比 $\mathrm{AD}$ 患者更 多的治疗选择, 包括药物治疗和手术治疗。大多数 PD 患者早期选择多巴胺替代药物治疗, 通过替代 系统中丢失的多巴胺, 减少运动症状。但随着使用 时间的延长, 其疗效逐渐下降, 并产生运动障碍等 副作用。脑深部电刺激疗法 (DBS), 又称为脑起 搏器植入术, 是目前常用的治疗晚期 PD 的手术疗 法, 可纠正由于多巴胺丧失而引起的基底节回路功 能异常, 帮助控制 PD 的运动症状。虽然 DBS 具 有微创、副作用较小等优点, 但手术费用昂贵, 存 在感染风险, 需要终生维护, 难以惠及众多的 PD 患者。因此, 仍需开发能够广泛推广的、无创的物 理治疗模式, 以应对我国老龄化人口的快速增长和 $\mathrm{PD}$ 病人的增加。

近年来, 澳大利亚 Mitrofanis 教授小组在不 同模型中对 PBM 治疗 PD 进行了深入的研究, 研 究结果发现光疗不仅能够缓解 PD 症状, 并且可 以保护神经细胞, 甚至能够通过外周组织远程光 疗抵抗 PD 的发生及进展。在 MPTP 诱导的 PD 小 鼠中, 非接触近距离 PBM (670 nm LED) 照射头 部, 能够保护黑质致密部 ( SNc) 中的多巴胺细胞 免受 MPTP 引起的毒性, 改善小鼠自主活动。并且 当 MPTP 剂量增加时, 光照剂量随之增加才能抵 抗 MPTP 引起的毒性 [29 31]。在 6-OHDA 损伤诱 导的 PD 大鼠中, PBM (670 nm LED) 能够有效 增加神经细胞存活率, 改善大鼠运动行为 [32]。在 K369I PD 小鼠中, PBM (670 nm LED) 使过度磷 酸化 tau 蛋白、NFTs 和氧化应激标志物（4- 羟基 壬烯醛和 8-羟基 - 2 - 脱氧鸟苷) 在近皮层和海马中 接近野生型水平, 并在存活神经元中恢复线粒体标
记细胞色素 Cox 的表达 [33]。而 $810 \mathrm{~nm} \mathrm{PBM}$ 治疗 也能改善 MPTP 诱导的 PD 小鼠的自主活动 [34]。 该小组设计了一种外科植入的乑内光纤, 分别将 $670 \mathrm{~nm}$ LED 或 $670 \mathrm{~nm}$ 激光送入 MPTP 治疗小鼠 的侧脑室, 两种治疗方式均能有效地保存 SNc 细 胞 [35]。随后, 该小组研究了 PBM 治疗对 MPTP 诱导的猴子 PD 模型的作用。通过乑内光纤 PBM $(670 \mathrm{~nm}, 25 \mathrm{~J}$ 和 $35 \mathrm{~J}$ ) 治疗, 降低了（约 75\%) MPTP 诱导的 SNc 和纹状体星形胶质细胞增生, 增 加了纹状体酪氨酸着化酶阳性 ( TH+) 细胞数量, 增加了胶质源性神经营养因子的表达 (GDNF), 改 善了猴子的 PD 临床症状 (体位、全身活动、运动 迟缓和面部), 但高剂量 PBM (670 nm, $125 \mathrm{~J}$ ) 治 疗并未引起猴子 PD 临床症状明显的改善 [36,37]。

Mitrofanis 教授小组研究发现 PBM 照射具有远 端效应, 通过光照射外周组织也能诱导神经保护作 用, 并将其称为远程 PBM。在 MPTP 诱导的 PD 小 鼠中, PBM (670 nm LED) 照射 BLAB/c 小鼠背部, 同时用铝䇴遮住头部, 能够减轻 $\mathrm{SNc}$ 多巴胺能细胞 的丢失 [38]。该小组在不同品系的小鼠 (C57BL/6) 中验证了这一结果, PBM (670 nm LED) 照射背部 后立即注射 MPTP, 6 天后检测显示多巴胺能细胞 的丢失减轻 [39]。但由于 PBM 治疗和 MPTP 注射 同时进行, 因此不确定 PBM 是通过远端治疗直接 影响了脑部神经功能, 还是影响了 MPTP 的药物代 谢动力学, 从而延缓了 PD 发生。该小组最新的研 究利用 PBM (670 nm LED) 照射小鼠背部和后肢, 10 天后注射 MPTP, 结果发现尽管没有直接照射头 部, 外周组织预处理 PBM 能明显减轻 MPTP 诱导 的中脑酪氨酸差化酶阳性多巴胺能细胞的丢失, 减 轻尾壳核复合体 FOS 阳性神经元的增加 [40]。从而 证实了 PBM 照射外周组织能够预防 MPTP 诱导的 PD 损伤, 明确了远端 PBM 的神经保护功能。因此 远程 PBM 预处理有望作为一种可行的神经保护干 预措施, 但其影响脑神经功能的作用机制尚不清楚。

光疗缓解 PD 的临床试验也在开展。西班牙 Santos 等 [41] 通过 $670 \mathrm{~nm}$ LED 经颖骨照射病人头 部 SNc 治疗 17 例 PD 患者, 对比对照组（17 例） 显示 PBM 治疗显著改善患者步态。一些学者研究 了白光照射对 $\mathrm{PD}$ 病人的作用, 每天两次在不同时 间段 (从 1 周到几个月) 使用强度和持续时间分别 为 1000 10 000 lux 和 0.5 1.5 h 的明亮光脉冲。德 
国 Paus 和澳大利亚 Willis 医生等报道白光治疗可逐 步改善 PD 患者相关的运动和精神参数 $[42,43]$ 。美 国 Videnovic 和荷兰 Rutten 医生报道白光治疗可改 善 PD 患者的睡眠质量, 有效缓解睡眠障碍 $[44,45]$ 。

\section{（三）弱光治疗与正常脑组织功能}

虽然许多研究已经证实 PBM 对神经疾病受试 者认知和记忆保持有积极作用, 但较少有研究探 讨 PBM 对健康受试者的认知功能。美国 Lima 教授 等 $[46,47]$ 测试了 tPBM 对 40 名本科生的记忆和注 意力的影响。受试者通过非接触 $1064 \mathrm{~nm}$ 激光对大 脑皮层的右额叶进行真假 PBM 照射。两周后, 延 迟匹配样本记忆任务显示, 与对照组相比, 治疗组 的记忆恢复延迟和正确试验次数有显著改善。积极 和消极情感计划（PANAS-X）追踪自我报告显示, 虽然参与者通常报告的积极情绪状态多于消极情绪 状态, 但与对照组相比, 治疗组具有更持续的积极 情绪状态, 总体情绪显著改善。这些数据表明, 经 颅激光刺激可以作为一种非侵入性和有效的方法来 增强大脑功能, 如与认知和情感维度有关的功能。 伊朗 Salimi 教授团队研究了 tPBM 对健康青年脑电 生理特征及注意力功能的影响 [48]。受试者通过右 前额叶皮层上的 $850 \mathrm{~nm}$ LED 接收真假 PBM 照射。 定量脑电图（qEEG）诊断显示 $\mathrm{tPBM}$ 后皮层脑电活 动发生改变, 对注意功能有明显影响。国内 Song P 课题组研究发现了 tPBM 对人运动皮层兴奋性的影 响 [49]。经颅 $820 \mathrm{~nm}$ 激光照射左侧初级运动皮层 和右侧第一背侧骨间肌, 经㐿磁刺激测量发现运动 诱发电位振幅显著增强, 表明 PBM 可短暂增加运 动皮层兴奋性。

目前大量研究表明 PBM 可以有效改善神经退 行性疾病模型小鼠和病人的认知、记忆和活动能力, 且并未观察到明显的副作用。大多数评估 PBM 治 疗神经退行性疾病的研究都是在进展的早期阶段进 行的, 并且表明, 与其他治疗方法一样, PBM 不 能挽救已经发生退行性病变的神经元, 使其免于调 亡或恢复到正常的细胞状态。所以, PBM 可以在 患者发病早期进行干预治疗, 及时遏制病情发展。 另一方面, PBM 的许多效果, 包括减少氧化应激 和增加血流, 都适用于更广泛的人群。总的来说, PBM 对健康患者有积极影响是合乎逻辑的。因此, 非侵入性 PBM 作用于正常人, 可有助于认知记忆
的改善, 提前干预, 延缓疾病发生。因此, 非侵入 性的 PBM 不仅可以发展为疾病无创治疗的有效手 段, 而且有望发展为 “治未病” 的无创理疗手段, 并具有低成本、易操作的特点, 便于广泛推广应用。

\section{三、光学治疗亟待突破的难题}

光疗经过几十年的发展, 已经证实在多种疾病 治疗中均有益处, 且越来越多的严格的科学研究也 证实了这一结论, 但该领域仍处于起步阶段, 很多 问题尚未明确, 首先是作用机制不清楚, 而且所报 道的治疗模式各异, 量效关系不清, 临床疗效证据 不足, 从而限制其发展。因此, 神经退行性疾病的 光学治疗亟待深入研究, 适用于临床的新技术手段 急需开发。

\section{（一）阐明弱光作用机理及量效关系}

在弱激光神经保护的机制研究中, 国外的 Wong-Riley 教授团队和 Hamblin 教授团队主要聚焦 于 $808 \mathrm{~nm}$ PBM 通过调节 CCO 活性增加 ATP 产量 的机制研究, 但相关研究并不深入。国内邢达教授 团队多年来研究 $633 \mathrm{~nm}$ PBM 通过细胞表面非受体 酪氨酸激酶对神经细胞信号转导通路的调节, 形成 了较完善的研究体系。但以上研究均是在细胞层 面进行, 缺少活体治疗中 PBM 的系统作用机制。 目前报道的不同 PBM 作用方式均能有效延缓神经 退行性疾病, 如颖内照射、血管照射、亮光经眼 睛照射, 但系统作用机制均不明确。特别是远程 PBM 的提出, 通过光照射外周组织如何作用于大 脑以诱导神经保护, 是否存在最佳的靶组织 / 器官 作为远程 PBM 的作用靶点及作用方式等。对于这 一新兴的研究领域, 仍有许多未回答的问题。因 此, 尚需深入探讨激光与组织相互作用的初始反 应过程，明确不同波长激光和不同作用模式下的 作用机制, 才能进一步指导光疗技术的发展和临床 应用。

弱光治疗的最大特点是无创性, 其功率密度通 常在毫瓦量级, 从 $1 \mathrm{~mW} / \mathrm{cm}^{2}$ 到 $100 \mathrm{~mW} / \mathrm{cm}^{2}$, 总 能量从 $1 \mathrm{~J}$ 到 $10 \mathrm{~J}$ 不等, 引起的局部温度升高不超 过 $0.1 \sim 0.75{ }^{\circ} \mathrm{C}$ 。越来越多的研究发现弱光特性的不 同会产生不同的生物学效应, 包括光剂量、光波长 及光的作用模式 (连续和脉冲)。另一方面, 在临 
床治疗过程中, 弱光的穿透深度是一个很重要的考 量指标。使用人类尸检样本进行的测量已经确定, 红光到近红外光穿过人类头皮和头骨的穿透范围在 $1 \% \sim 3 \%$, 不到 $0.03 \%$ 的发射光能穿透 $12 \mathrm{~mm}$ 的脑 组织 [50]。因此, 明确对于特定区域调控的最优参 数特征, 包括适用于人体的最佳作用波长、最佳作 用模式 (脉冲或连续)、最佳剂量及最佳治疗方式(颖 外、经颅、鼻腔或视觉照射), 对于临床应用及推 广具有重要的指导意义。

\section{（二）开发新型弱光治疗技术}

目前国内外市场开发的用于神经退行性疾病 的光疗设备, 波长主要以红光和近红外光为主, 模式主要为便携式鼻腔照射仪和头盔光疗仪。包 括 $670 \mathrm{~nm}$ 半导体连续激光鼻内治疗仪, $810 \mathrm{~nm}$ (10 Hz) 脉冲 LED 鼻内 / 颅内治疗仪等。随着医学 治疗模式的转变和弱光治疗的疾病谱从常见病向重 大慢性增龄性疾病的拓展, 弱光的治疗领域正在经 历从疾病治疗到疾病预防的延伸, 其应用的主战场 也正逐渐从医疗机构向社区和家庭倾斜, 这就对弱 光治疗设备的便携、小型化、微型化及可穿戴化等 提出了更高的要求。法国科学家开发出一种激光针 织面料, 可以通过光照射治疗皮炎, 有望实现可穿 戴、无痛无副作用的治疗模式。通过将蓝光耦合到 包裹在织物中的直径是 $160 \mu \mathrm{m}$ 的聚合物光纤中, 形成的可穿戴光子可用于治疗新生儿黄㾝, 获得了 美国 FDA 批准的蓝光可穿戴设备可用于治疗轻度 银屑病等。随着光电子技术的快速发展, 开发适 用于神经退行性疾病治疗的便携式、小型化、可 穿戴设备具有重大的意义和市场前景。另一方面, 随着可穿戴设备的应用, 需要实时监测技术和大 数据平台的支撑。

\section{（三）完善临床验证体系及评价指标}

尽管国内外均有临床试验显示弱光疗法可改 善神经退行性疾病, 认为其能够成为具有潜力的无 创治疗手段, 但由于各研究组使用不同光参数和治 疗模式, 不同治疗组之间疗效无法互相验证, 仍需 进一步证实, 最终难以形成统一的可推广的临床治 疗方案。建立光疗的临床验证体系和评价指标, 才 能有效验证不同光疗参数的治疗效果, 评价其可行 性。有助于明确适用于临床神经退行性疾病的治疗
模式, 快速评价光疗设备的有效性, 从而保障治疗 技术的广泛应用和发展, 保障治疗设备的快速转化 和推广。另一方面, 构建大数据云平台, 及时获得 病人信息, 便于远程指导治疗, 从而实现个性化治 疗模式, 保障和促进可穿戴医疗发展。

图 2 展示了光学治疗几个努力方向之间的关系。 首先, 要明确光疗为什么会有如此广谱的神经保护 效应, 以及光是如何调控机体达到神经保护作用, 对光疗机制的理解才能从根本上推动光疗的应用。 其次, 目前研究中的光源类型、光疗参数及作用方 式各有不同, 明确量效关系是保障光疗临床推广的 关键所在。同时, 需要更广泛、深入的临床试验, 明确临床可用的治疗模式, 针对不同疾病开发便携 的治疗设备, 完善临床评价体系。最后建立大数据 云监测智能平台, 科学监控并远程指导治疗, 保障 便携式和可穿戴设备的安全性、有效性。

\section{四、结语}

相关研究已经在不同动物模型和病人中验证 $\mathrm{PBM}$ 对 $\mathrm{AD} 、 \mathrm{PD}$ 等的疗效, 表明 $\mathrm{PBM}$ 可能是未来 治疗这些疾病的一种有效的替代疗法。由于 PBM 相对无副作用的优点, 它还可以作为当前有效治疗 的辅助疗法, 甚至可能发展为一种预防性理疗手段, 总的来说, 光疗在脑疾病的治疗和防治中是非常有 前景的。但光疗要成为一种广泛可及的治疗方法, 目前的动物实验和小型临床验证是远远不够的, 只

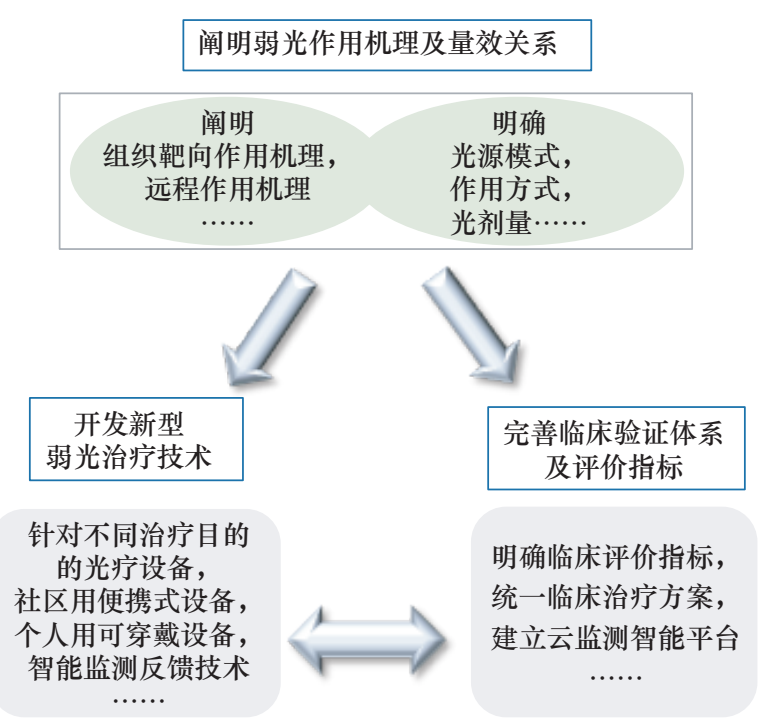

图 2 弱光疗法的潜在研究方向及相关性 
有把阐明组织靶向作用机理、远程作用机理，开发 新型弱光治疗技术，完善临床验证等体系及评价指 标等几个方向相互协作, 共同发展, 才能促进光学 疗法的临床应用和推广，尽快造福病患，服务社会。

\section{参考文献}

[1] Yun S H, Kwok S J J. Light in diagnosis, therapy and surgery [J]. Nature Biomedical Engineering, 2017, 1(1): 1-16.

[2] Hong N. Photobiomodulation as a treatment for neurodegenerative disorders: Current and future trends [J]. Biomedical Engineering Letters, 2019, 9(3): 359-366.

[3] Hamblin M R. Shining light on the head: Photobiomodulation for brain disorders [J]. BBA Clinical, 2016, 6: 113-124.

[4] Hamblin M R. Mechanisms and mitochondrial redox signaling in photobiomodulation [J]. Photochemistry and Photobiology, 2018, 94(2): 199-212.

[5] Meng C, He Z, Xing D. Low-level laser therapy rescues dendrite atrophy via upregulating BDNF expression: Implications for Alzheimer's disease [J]. Journal of Neuroscience, 2013, 33(33): 13505-13517.

[6] Zhang H, Wu S, Xing D. Inhibition of A 325 -35-induced cell apoptosis by Low-power-laser-irradiation (LPLI) through promoting Akt-dependent YAP cytoplasmic translocation [J]. Cellular Signalling, 2012, 24(1): 224-32.

[7] Liang J, Liu L, Xing D. Photobiomodulation by low-power laser irradiation attenuates $A \beta$-induced cell apoptosis through the Akt/GSK3 $\beta / \beta$-catenin pathway [J]. Free Radical Biology and Medicine, 2012, 53(7): 1459-1467.

[8] Song S, Zhou F, Chen W R. Low-level laser therapy regulates microglial function through Src-mediated signaling pathways: Implications for neurodegenerative diseases [J]. Journal of Neuroinflammation, 2012, 9(1): 219.

[9] Birks J S. Cholinesterase inhibitors for Alzheimer's disease [J]. Cochrane database of systematic reviews, 2006, 1(1): CD005593.

[10] Kishi T, Matsunaga S, Iwata N. The effects of memantine on behavioral disturbances in patients with Alzheimer's disease: A meta-analysis [J]. Neuropsychiatric Disease and Treatment, 2017, 13: 1909 .

[11] Taboada L D, Yu J, El-Amouri S, et al. Transcranial laser therapy attenuates amyloid- $\beta$ peptide neuropathology in amyloid- $\beta$ protein precursor transgenic mice [J]. Journal of Alzheimer's Disease, 2011, 23(3): 521-535.

[12] Tao L, Wang X, Zhou Q, et al. Near infra-red light treatment of Alzheimer's disease [C]. Bellingham: International Society for Optics and Photonics, 2019.

[13] Purushothuman S, Johnstone D M, Nandasena C, et al. Photobiomodulation with near infrared light mitigates Alzheimer's disease-related pathology in cerebral cortex-evidence from two transgenic mouse models [J]. Alzheimer's Research \& Therapy, 2014, 6(1): 2 .

[14] Farfara D, Tuby H, Trudler D, et al. Low-level laser therapy ameliorates disease progression in a mouse model of Alzheimer's disease [J]. Journal of Molecular Neuroscience, 2015, 55(2): 430-436.
[15] Comerota M M, Krishnan B, Taglialatela G. Near infrared light decreases synaptic vulnerability to amyloid beta oligomers [J]. Scientific Reports, 2017, 7(1): 1-11.

[16] Lu Y, Wang R, Dong Y, et al. Low-level laser therapy for beta amyloid toxicity in rat hippocampus [J]. Neurobiology of Aging, 2017, 49: 165-182.

[17] Grillo S L, Duggett N A, Ennaceur A, et al. Non-invasive infra-red therapy $(1072 \mathrm{~nm})$ reduces $\beta$-amyloid protein levels in the brain of an Alzheimer's disease mouse model, TASTPM [J]. Journal of Photochemistry and Photobiology B: Biology, 2013, 123: 13-22.

[18] Zinchenko E, Navolokin N, Shirokov A, et al. Pilot study of transcranial photobiomodulation of lymphatic clearance of betaamyloid from the mouse brain: Breakthrough strategies for nonpharmacologic therapy of Alzheimer's disease [J]. Biomedical Optics Express, 2019, 10(8): 4003-4017.

[19] Zhang Y, Wang F, Luo X, et al. Cognitive improvement by photic stimulation in a mouse model of Alzheimer's disease[J]. Current Alzheimer Research, 2015, 12(9): 860-869.

[20] Iaccarino H F, Singer A C, Martorell A J, et al. Gamma frequency entrainment attenuates amyloid load and modifies microglia [J]. Nature, 2016, 540(7632): 230-235.

[21] Adaikkan C, Middleton S J, Marco A, et al. Gamma entrainment binds higher-order brain regions and offers neuroprotection $[\mathrm{J}]$. Neuron, 2019, 102(5): 929-943.

[22] Martorell A J, Paulson A L, Suk H-J, et al. Multi-sensory gamma stimulation ameliorates Alzheimer's-associated pathology and improves cognition [J]. Cell, 2019, 177(2): 256-271, e22.

[23] 金丽英, 石秉霞, 周畅. 半导体激光疗法对轻度认知障碍病人 $\beta$-类淀粉样蛋白的影响 [J]. 青岛大学医学院学报, 2000, 36(3): 175-176.

Jin L Y, Shi B X, Zhou C. The effect on serum amyloid $\beta$ protein of patients with mild cognitive impairment after semiconductor laser therapy [J]. Journal of Qingdao University (Medical Sciences), 2000, 36(3): 175-176.

[24] Arakelyan H S. Treatment of Alzheimer's disease with a combination of laser, magnetic field and chromo light (colour) therapies: A double-blind controlled trial based on a review and overview of the etiological pathophysiology of Alzheimer's disease [J]. Laser Therapy, 2005, 14(1): 19-28.

[25] Maksimovich I V. Dementia and cognitive impairment reduction after laser transcatheter treatment of Alzheimer's disease [J]. World Journal of Neuroscience, 2015, 5(3): 189.

[26] Lim L. The potential of treating Alzheimer's disease with intranasal light therapy [C]. Toronto: MedicLights Research Inc., 2013.

[27] Berman M H, Halper J P, Nichols T W, et al. Photobiomodulation with near infrared light helmet in a pilot, placebo controlled clinical trial in dementia patients testing memory and cognition [J]. Journal of Neurology and Neuroscience, 2017, 8(1): 176.

[28] Saltmarche A E, Naeser M A, Ho K F, et al. Significant improvement in cognition in mild to moderately severe dementia cases treated with transcranial plus intranasal photobiomodulation: case series report $[\mathrm{J}]$. Photomedicine and Laser Surgery, 2017, 35(8): 432-441.

[29] Shaw V E, Peoples C, Spana S, et al. Patterns of cell activity in the subthalamic region associated with the neuroprotective 
action of near-infrared light treatment in MPTP-treated mice [J]. Parkinson's Disease, 2012, $2012: 296875$.

[30] Moro C, Torres N, Massri N E, et al. Photobiomodulation preserves behaviour and midbrain dopaminergic cells from MPTP toxicity: Evidence from two mouse strains [J]. BMC Neuroscience, 2013, 14(1): 40.

[31] Massri N E, Johnstone D M, Peoples C L, et al. The effect of different doses of near infrared light on dopaminergic cell survival and gliosis in MPTP-treated mice [J]. International Journal of Neuroscience, 2016, 126(1): 76-87.

[32] Reinhart F, Massri N E, Chabrol C, et al. Intracranial application of near-infrared light in a hemi-parkinsonian rat model: The impact on behavior and cell survival [J]. Journal of Neurosurgery, 2016, 124(6): 1829-1841.

[33] Purushothuman S, Nandasena C, Johnstone D M, et al. The impact of near-infrared light on dopaminergic cell survival in a transgenic mouse model of parkinsonism [J]. Brain Research, 2013, 1535: 61-70.

[34] Reinhart F, Massri N E, Darlot F, et al. $810 \mathrm{~nm}$ near-infrared light offers neuroprotection and improves locomotor activity in MPTPtreated mice [J]. Neuroscience Research, 2015, 92: 86-90.

[35] Moro C, Massri N E, Torres N, et al. Photobiomodulation inside the brain: A novel method of applying near-infrared light intracranially and its impact on dopaminergic cell survival in MPTP-treated mice [J]. Journal of Neurosurgery, 2014, 120(3): 670-683.

[36] Darlot F, Moro C, Massri N E, et al. Near-infrared light is neuroprotective in a monkey model of Parkinson disease [J]. Annals of Neurology, 2016, 79(1): 59-75.

[37] Massri N E, Lemgruber A P, Rowe I J, et al. Photobiomodulationinduced changes in a monkey model of Parkinson's disease: Changes in tyrosine hydroxylase cells and GDNF expression in the striatum [J]. Experimental Brain Research, 2017, 235(6): 1861-1874.

[38] Johnstone D M, El Massri N, Moro C, et al. Indirect application of near infrared light induces neuroprotection in a mouse model of parkinsonism-An abscopal neuroprotective effect [J]. Neuroscience, 2014, 274: 93-101.

[39] Kim B, Mitrofanis J, Stone J, et al. Remote tissue conditioning is neuroprotective against MPTP insult in mice [J]. Ibro Reports,
2018, 4: 14-17.

[40] Ganeshan V, Skladnev N V, Kim J Y, et al. Pre-conditioning with remote photobiomodulation modulates the brain transcriptome and protects against MPTP insult in mice [J]. Neuroscience, 2019, 400: 85-97.

[41] Santos L, Olmo-Aguado S D, Valenzuela P L, et al. Photobiomodulation in Parkinson's disease: A randomized controlled trial [J]. Brain Stimulation, 2019, 12(3): 810.

[42] Willis G L, Boda J, Freelance C B. Polychromatic light exposure as a therapeutic in the treatment and management of Parkinson's disease: A controlled exploratory trial [J]. Frontiers in Neurology, 2018, 9: 741.

[43] Paus S, Schmitz-Hübsch T, Wüllner U, et al. Bright light therapy in Parkinson's disease: A pilot study [J]. Movement Disorders: Official Journal of the Movement Disorder Society, 2007, 22(10): $1495-1498$.

[44] Videnovic A, Klerman E B, Wang W, et al. Timed light therapy for sleep and daytime sleepiness associated with Parkinson disease: A randomized clinical trial [J]. Jama Neurology, 2017, 74(4): 411-418.

[45] Rutten S, Vriend C, Smit J H, et al. Bright light therapy for depression in Parkinson disease: A randomized controlled trial [J]. Neurology, 2019, 92(11): e1145-e1156.

[46] Gonzalez-Lima F, Barrett D W. Augmentation of cognitive brain functions with transcranial lasers [J]. Frontiers in Systems Neuroscience, 2014, 8: 36.

[47] Barrett D W, Gonzalez-Lima F. Transcranial infrared laser stimulation produces beneficial cognitive and emotional effects in humans [J]. Neuroscience, 2013, 230: 13-23.

[48] Jahan A, Nazari M A, Mahmoudi J, et al. Transcranial near-infrared photobiomodulation could modulate brain electrophysiological features and attentional performance in healthy young adults [J]. Lasers in Medical Science, 2019, 34(6): 1193-1200.

[49] Song P, Han T, Lin H, et al. Transcranial near-infrared stimulation may increase cortical excitability recorded in humans [J]. Brain Research Bulletin, 2020, 155: 155-158.

[50] Hart N S, Fitzgerald M. A new perspective on delivery of rednear-infrared light therapy for disorders of the brain [J]. Discovery Medicine, 2016, 22(120): 147-156. 